\title{
Clinical effectiveness of orthodontic miniscrew implantation guided by a novel cone beam CT image-based computer aided design and computer aided manufacturing (CAD-CAM) template
}

\author{
Lingling Qiu ${ }^{1}$, Hui Xu ${ }^{1}$, Pingping Feng ${ }^{1}$, Xiaoyan Sha ${ }^{2}$, Hongwei Zhang ${ }^{3}$ \\ ${ }^{1}$ Orthodontic Department, School of Stomatology, Capital Medical University, Beijing, China; ${ }^{2}$ Radiology Department, School of Stomatology, \\ Capital Medical University, Beijing, China; ${ }^{3}$ School of Mechanical Engineering, Beijing Institute of Petrochemical Technology, Beijing, China \\ Contributions: (I) Conception and design: L Qiu; (II) Administrative support: L Qiu, P Feng; (III) Provision of study materials or patients: L Qiu, \\ H Zhang, P Feng; (IV) Collection and assembly of data: L Qiu, X Sha; (V) Data analysis and interpretation: H Xu; (VI) Manuscript writing: All \\ authors; (VII) Final approval of manuscript: All authors. \\ Correspondence to: Dr. Lingling Qiu, PhD. Orthodontic Department, School of Stomatology, Capital Medical University. 4 Tiantan Xili, Dongcheng \\ District, Beijing 100050, China. Email: q1199@126.com.
}

Background: Although miniscrews are widely used in orthodontic treatment as temporary anchorage devices, their correct and safe placement has attracted little attention. This study aimed to introduce a novel cone beam CT (CBCT) image-based computer aided design and computer aided manufacturing (CAD-CAM) template for orthodontic miniscrew implantation and to evaluate the effectiveness of miniscrews implanted under the guidance of this template.

Methods: The CBCT scans of ten patients requiring miniscrews as anchorage were analyzed in NNT software to predetermine the insertion sites of miniscrew implants. The DICOM data of the scans, along with virtual miniscrews acquired in Solidworks software, were imported into Mimics software to construct three-dimensional (3D) images of teeth and bone and to determine the virtual position of miniscrews and $3 \mathrm{D}$ virtual templates were designed following consideration of the virtual implantation plans. A STL (Stereolithography) file of the virtual template was output, and the resin template was then fabricated with a stereolithographic appliance (SLA). 24 Miniscrews were then implanted guided by the template and clinical evaluation of their safety and stability, as well as their placement deviations, were made. A dental casts model and cephalometric analysis before and after orthodontic treatment were made to assess the dentomaxillofacial changes.

Results: All 24 miniscrews had no contact with adjacent roots. 18 miniscrews had a grade I safety score and six had a grade II. The miniscrews were stable at 1, 3, 6, and 9 months after implantation, although there was mild inflammation around two miniscrews. Implantation deviation of miniscrew in the crown was $(1.03 \pm 0.65)$ and $(1.26 \pm 0.72) \mathrm{mm}$ in the apex, on average. Satisfactory dentomaxillofacial changes in 10 patients with these 24 miniscrews as anchorage were acquired.

Conclusions: Miniscrews could be implanted in the targeted position safely and precisely when guided by the novel templates, and remained stable during orthodontic treatment. Patients treated with these miniscrews as anchorage in orthodontic treatment acquired satisfactory dentomaxillofacial changes.

Keywords: Computer aided design and computer aided manufacturing (CAD-CAM); orthodontics; miniscrew; template

Submitted Apr 28, 2021. Accepted for publication Jun 04, 2021.

doi: $10.21037 / \mathrm{atm}-21-2575$

View this article at: https://dx.doi.org/10.21037/atm-21-2575 


\section{Introduction}

Miniscrews are widely used in orthodontic treatment as a temporary anchorage device for orthodontic treatment (1). The application of miniscrews reduces unwanted tooth movement and provides anchorages outside the dentition, which can form a new force system to treat more complicated orthodontic patients, and greatly expand the indications of orthodontic treatment.

Obtaining the required force system relies on the precise positioning of miniscrews. The insertion position is mainly determined based on two factors: the anatomical characteristics of the individuals dentomaxillofacial structure, and the treatment goal. After the exact insertion position is determined, efforts should be made to implant miniscrews precisely, safely, and stably.

However, "safe areas" on the upper and lower jaws suitable for miniscrew implantation are limited $(2,3)$. Insufficient cortical bone thickness (4) and a narrow attached gingival width (5) make this difficult, and damage to adjacent teeth, such as root and pulp injuries, are not uncommon $(6,7)$. Root damage may affect the longterm stability of miniscrews, and their fracture during implantation causes great trauma (8-10). Despite these complications and the $19.3 \%$ and $12 \%$ failure rate in mandible and maxilla miniscrews respectively (11), they remain a necessary temporary anchorage device for complicated orthodontic treatment.

Many efforts had been made to improve the stability and safety of miniscrews. Using radiographs, markers such as copper wire (12), 3-dimensional (3D) surgical guide (13), and $3 \mathrm{D}$ radiographic surgical guide (14) have been used, but these are all based on two-dimensional X-ray, which cannot reproduce the 3D dentomaxillofacial structure (15).

The application of digital technology in stomatology has improved the precision of oral treatment. Dentistry is transforming with a speed increasing trend towards digitalization. CAD-CAM, computer aided design and computer aided manufacturing, which use computer as a major part in design and manufacture of dental inlays, onlays, veneers, crowns, fixed partial dentures, implant abutments have become increasingly popular in dentistry. CBCT has provided more information on $3 \mathrm{D}$ dentomaxillofacial structure, and CAD-CAM surgical guides based on CBCT images have made the implantation process simpler and more precise. Previous studies using CBCT image based surgical stents for miniscrew implantation with the same process and method as a prosthetic implant template, found the accuracy of implantation guided by stents was improved (16). However, these guides are mainly used for prosthetic implants, and due to the different shape, size, insertion site, and intended use between prosthetic implants and orthodontic miniscrews, the complicated process of this guide construction makes it unsuitable for miniscrew implantation in orthodontic treatment. Another CAD-CAM surgical guide for the palatal miniscrew insertion was introduced, but the fusion of conebeam computed tomography (CBCT) and digital dental model images was needed to make this guide, which make the process complicated and increase the insertion deviation (17). More simple and suitable guides are required for the use of miniscrews, and in this study we modified surgical stents on the stent design and avoid the fusion process. A digital workflow of the design and fabrication of cone beam CT image-based CAD-CAM templates for orthodontic miniscrew implantation was introduced, and a clinical evaluation of template-guided-implanted miniscrews and treatment results was made.

We present the following article in accordance with the STROBE reporting checklist (available at https://dx.doi. org/10.21037/atm-21-2575).

\section{Methods}

The ethics committee of Beijing Stomatological Hospital approved the study protocol (2015 No. 55), which was conducted in full accordance with ethical principles, including the World Medical Association Declaration of Helsinki (as revised in 2013). All patients included in this study signed an informed consent for the scientific use of data.

Patients admitted to the Department of Orthodontics, of Beijing Stomatological Hospital between May 2015 and February 2018 were selected. Ten patients who were diagnosed by three orthodontists, each with more than 13 years of experience, as having malocclusion requiring miniscrew anchorage were included. Based on the treatment goal and CBCT evaluation of the expected insertion areas, implantation positions were predetermined. Some risks were anticipated, due to narrow root distance, insufficient buccal bone thickness, and low bottom of maxillary sinus in the implantation area in some patients. Cone-beam CT image based customized CAD-CAM templates were made, and $1.6 \mathrm{~mm}$ $\times 9 \mathrm{~mm}$ miniscrews (Ningbo Cibei Medical Equipment Co. LTD., China) were implanted under guidance of the templates. The deviation of miniscrews in the crown and apex were defined as the accuracy of implantation 


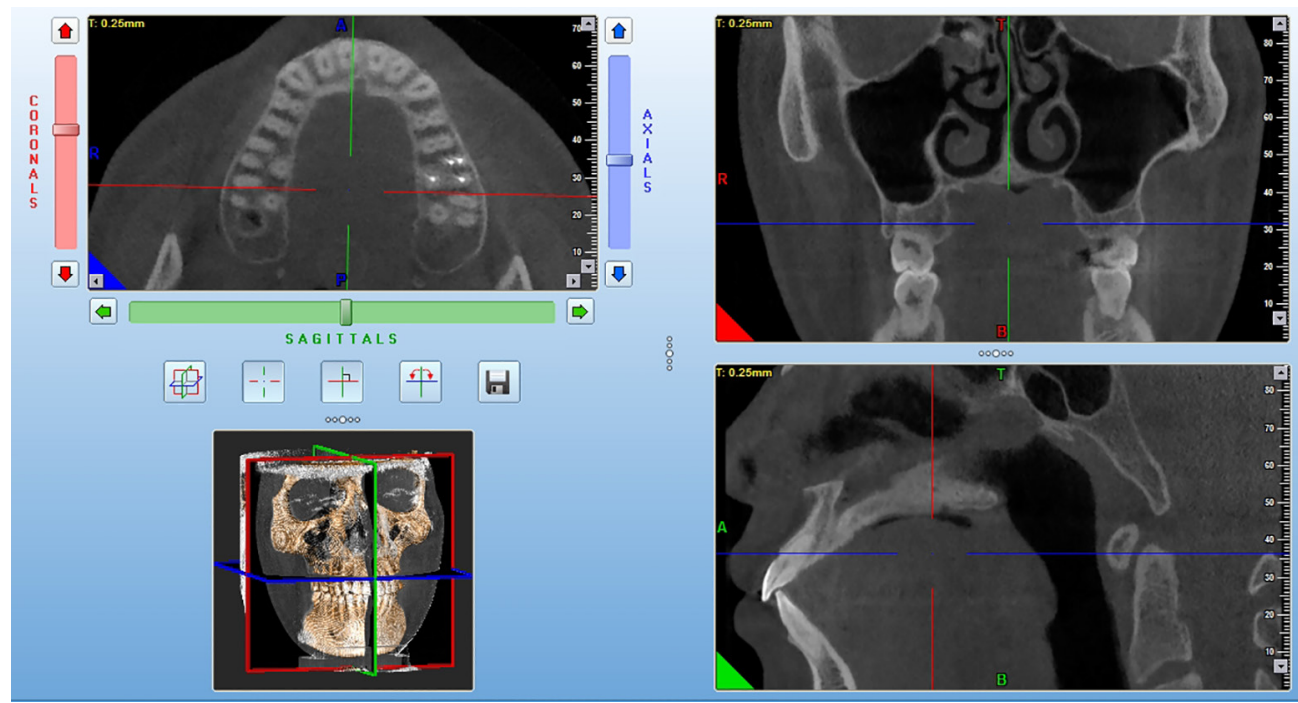

Figure 1 Analysis of maxillofacial structure in NNT software.

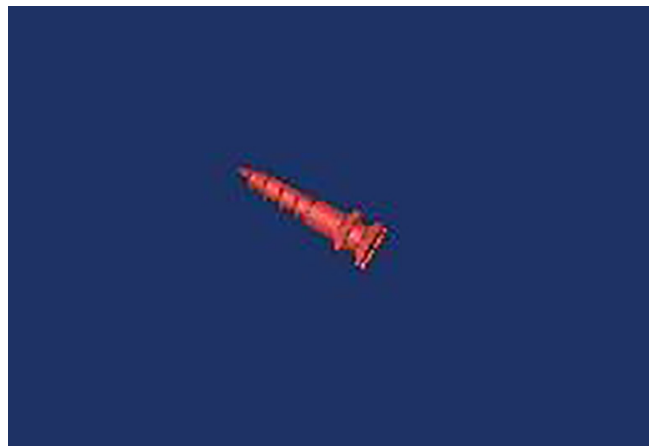

Figure 2 3D image of miniscrew.

and were measured. The failure rate and condition of the oral mucosa surrounding the miniscrews after 1, 3, 6 , and 9 months, were investigated. Facial and occlusion photographs and lateral cephalometric radiographs were taken, and cast models were made before and after treatment to analyze the treatment result with miniscrews as temporary anchorage.

\section{Workflow of design and fabrication of cone beam CT image-based CAD-CAM templates for orthodontic miniscrew implantation}

The maxillofacial region was scanned by cone beam CT (NewTom, VGI, Italy), and a 3D analysis of the anatomic structure was carried out in NNT software (Figure 1).

Three doctors confirmed the presence of anatomical factors that might affect the safety and stability of the miniscrews. The CBCT data of patients was then imported into Mimics 11.01 software (mimics 11.01, material, Belgium) to reconstruct 3D images of the teeth and jaws. The patients were instructed to bite $3 \mathrm{~mm}$ thick wax in the upper and lower anterior teeth during the CBCT scan to separate the occlusion and avoid overlapping of the occlusal surface. After 3D images of the miniscrews were drawn with Solidworks software (Figure 2), they were imported into mimics 11.01 software.

The position and direction of miniscrews were determined under consideration of the orthodontic purpose, local condition of the jaw, miniscrew shape, and other factors (Figure 3).

After the virtual miniscrew was placed in the jaw, its crown was extended using the 3D software drawing tool, and a cylinder which had the same direction of the virtual miniscrew was placed. The diameter of the cylinder could be changed to $0.5 \mathrm{~mm}$ larger than the maximum diameter of the screwdriver end (Figure 4).

The template was designed by layer-by-layer coating and Boolean operation (Figure 5A) in Mimics software, and a virtual template was designed (Figure $5 B$ ). The template comprised a retention part, a guide part, and a connecting part, and the former was fit to the teeth close to the insertion site. The guide part was tube matched with the cylinder end of the screwdriver which holds the miniscrew, and the length of the tube was $0.5-1.0 \mathrm{~cm}$. A space between the guide part and mucosa surface of the insertion site 


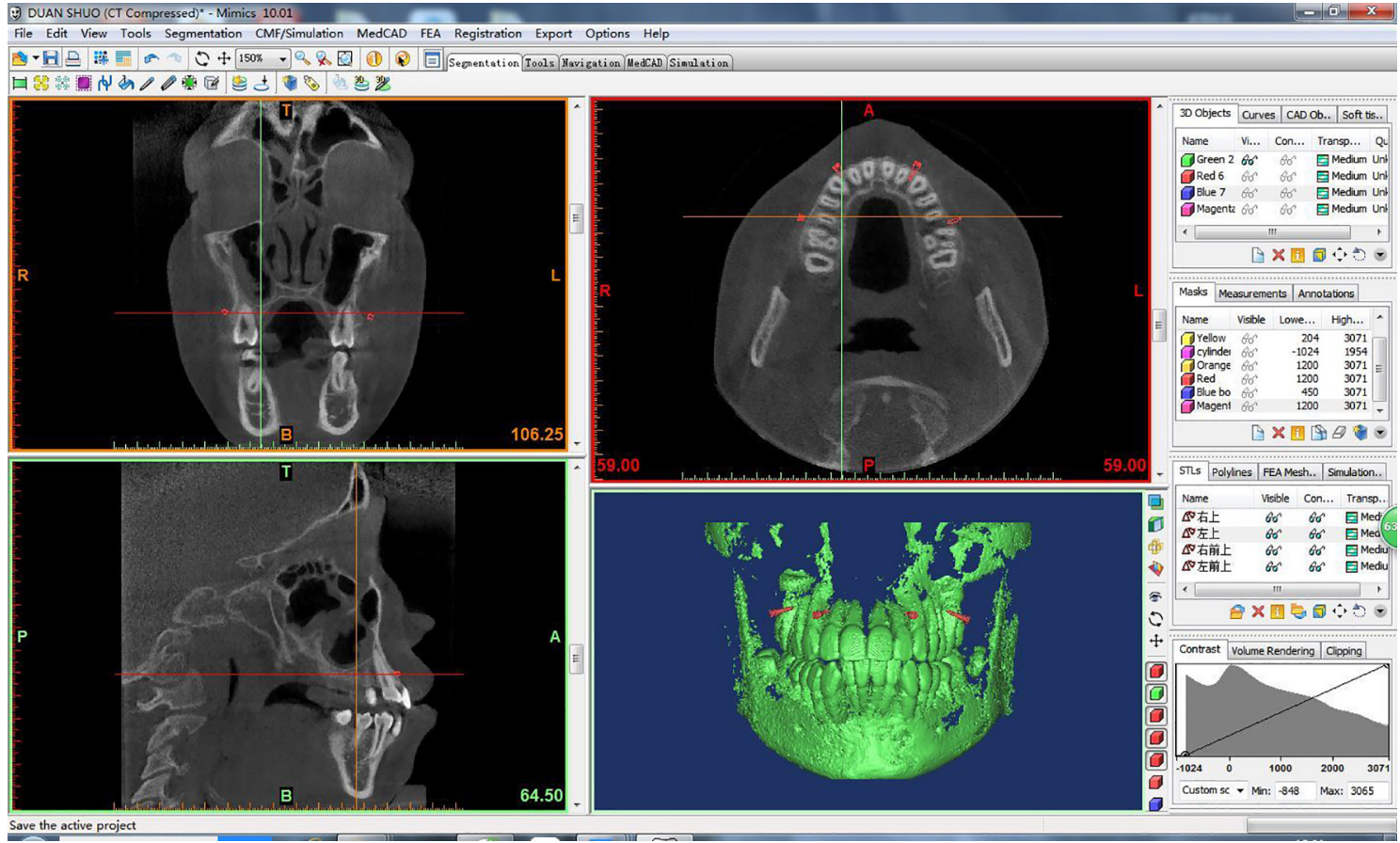

Figure 3 Virtual miniscrew placement.
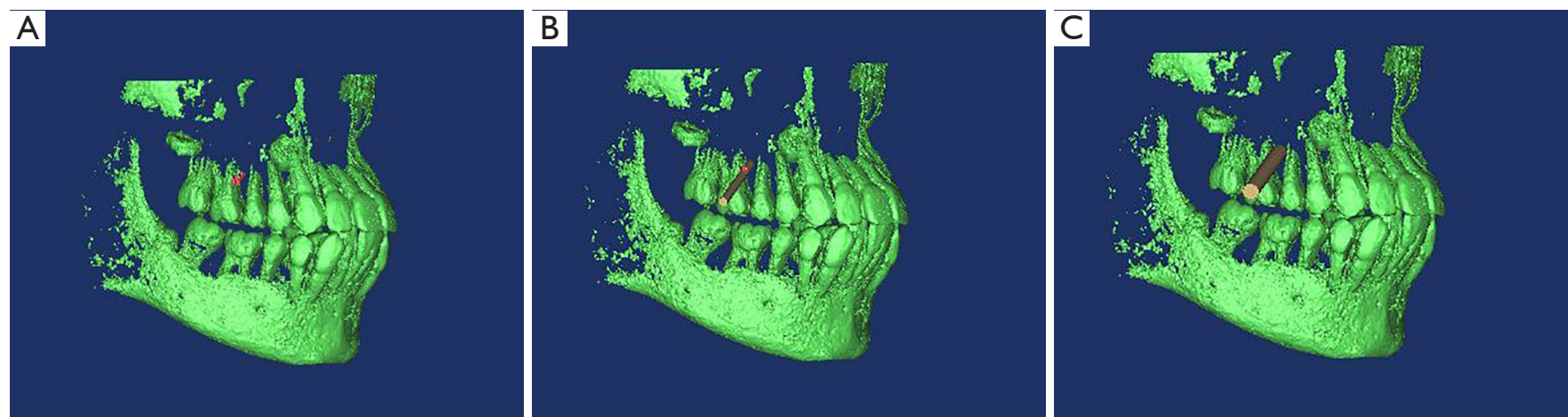

Figure 4 Implantation tunnel formation (implantation tunnel was formed from step A to step C). (A) Virtual miniscrew placed, (B) cylinder placed, (C) cylinder changed to $0.5 \mathrm{~mm}$ diameter larger than the maximum diameter of the screwdriver end.

ensured the implantation process was visible and the template could be easily removed afterwards. The space was made longer than the crown exposed part of the miniscrew and shorter than the cylinder end of the screwdriver, and the retention part and the guide part were connected by a narrow connecting part (Figure 5C).

The STL file of the virtual template was exported and printed into the resin template with a $3 \mathrm{D}$ rapid prototyping machine (3D pro, object lo. ITD, Israel) and checked for proper fit.

\section{Miniscrew implantation guided by template}

The template was placed on the teeth close to the insertion area. The retention part of the template was then fit into the crown of the teeth, light force was applied on the occlusal surface of the retention part to steady it, the cylinder tip placed on the screwdriver carrying the miniscrew was placed into the guide tube of the template, and the miniscrew then implanted along the guide tube (Figure 6). 

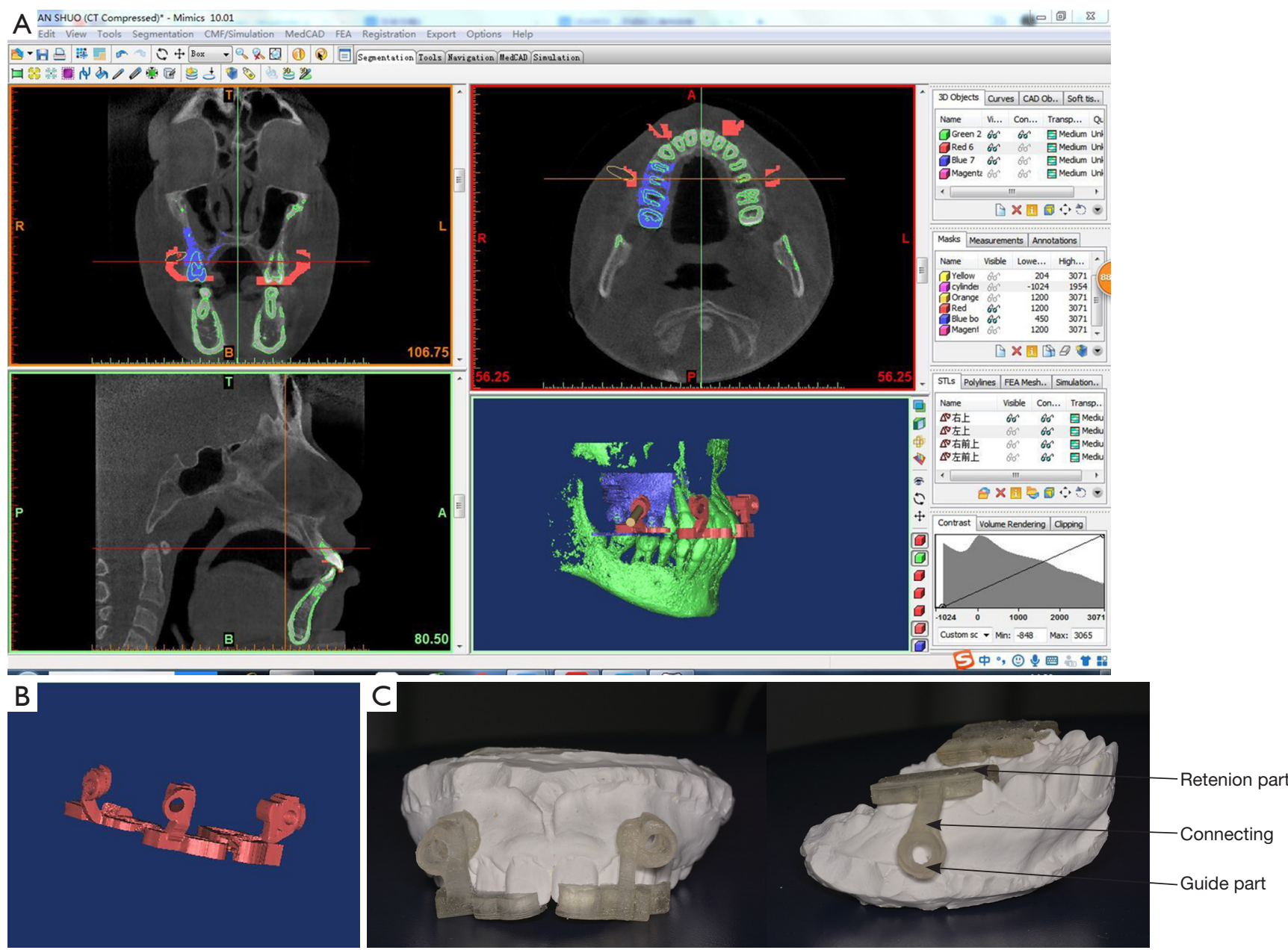

Figure 5 Design and fabrication of template. $(\mathrm{A}, \mathrm{B})$ Virtual template, $(\mathrm{C})$ resin template.

\section{Clinical examination of miniscrew}

The mobility of miniscrews was checked immediately after implantation, and 1, 3, 6, and 9 months after surgery, and divided into four levels: level I indicated no looseness; level II showed movement $\leq 0.5 \mathrm{~mm}$; level III movement $>0.5$ and $\leq 1.0 \mathrm{~mm}$; and level IV indicated movement $>1.0 \mathrm{~mm}$.

The conditions of the implant area, including plaque accumulation, mucosal swelling, and bleeding around the miniscrews, were recorded immediately after implantation, and at 1 month, 3 months, 6 months, and 9 months after implantation, and the failure rate and loss of miniscrews was recorded at the same time intervals.

Following surgery, a cone beam CT was taken to determine the relationship between the $3 \mathrm{D}$ position of the miniscrew and the adjacent tooth root. Before evaluation, a radiologist with 9 years of clinical work experience evaluated the image to ensure the image quality was good, with clearly displayed tooth root and bone, and no obvious metal artifact. The axial plane of the cone beam CT image with the closest distance between miniscrew and root was selected, and this distance was measured (Figure 7).

Implantation safety was classified as follows: Grade I: the distance between miniscrew and root was greater than or equal to $1 \mathrm{~mm}$; grade II the distance between implant and root was less than $1 \mathrm{~mm}$, but the miniscrew did not contact the root; grade III the miniscrew just contacted the root; grade IV the miniscrew contacted the tooth root but the contact area was less than the thickness of the root canal wall; grade $\mathrm{V}$ the miniscrew touched the root more than the thickness of the root canal wall; and grade VI the miniscrew penetrated the root. 

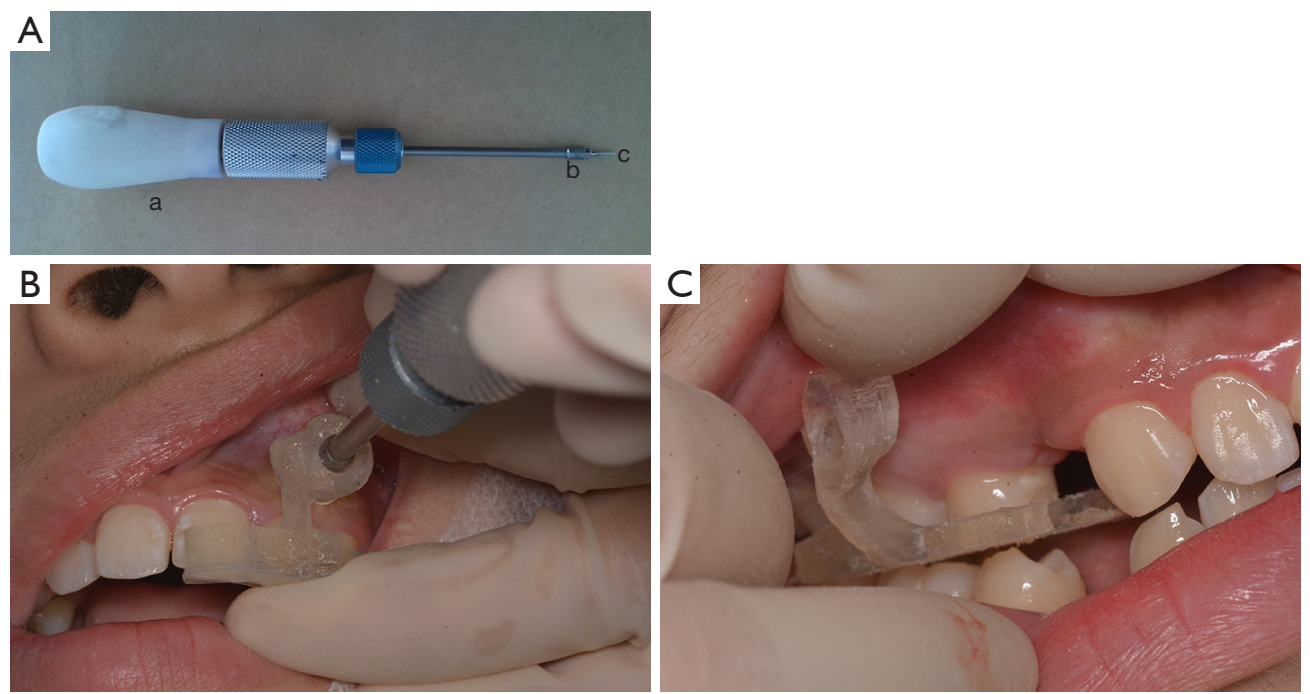

Figure 6 Miniscrew implantation under the guidance of template. (A) Screwdriver, (B) cylinder tip of screwdriver (C) miniscrew
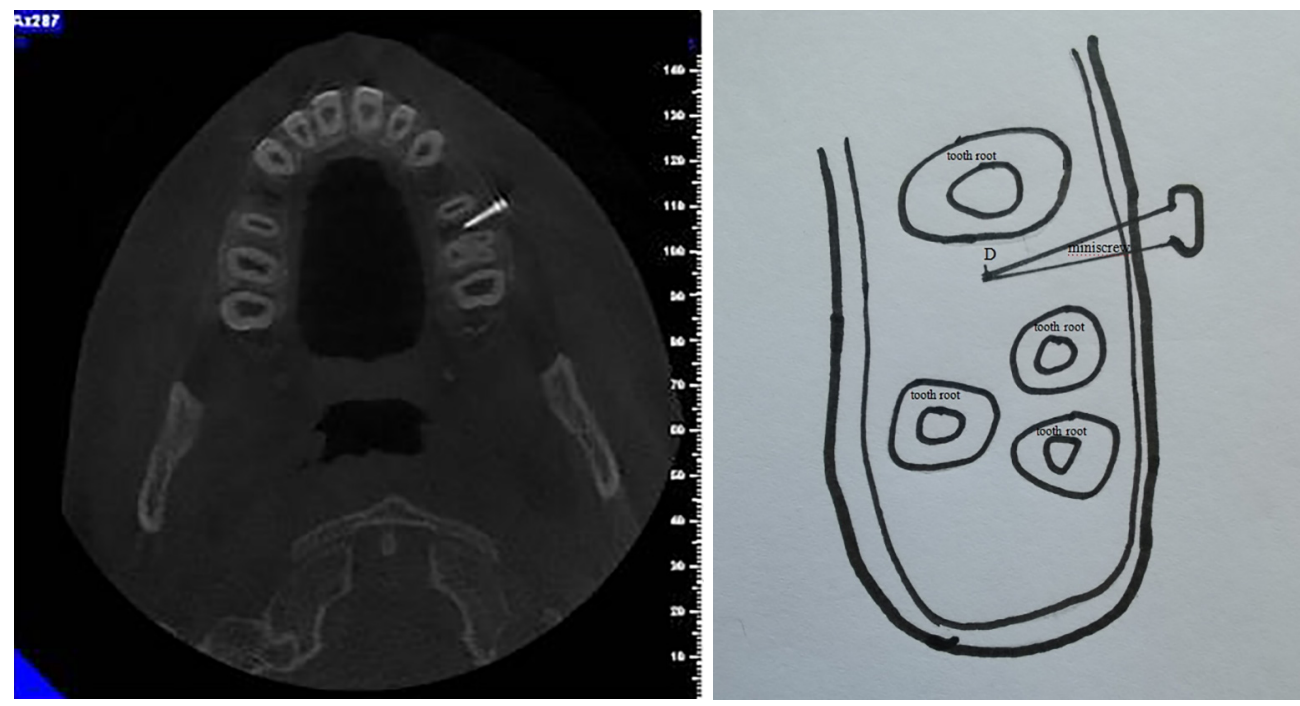

Figure 7 Implantation safety measurement. D, closest distance between miniscrew and tooth root.

Cone beam CT images before and after implantation were superimposed to evaluate implantation deviation. After the implantation, the DICOM data of the cone beam CT were imported into Mimics software and post-implantation CBCT images were superimposed on pre-implantation ones using 12 points (maxillary anterior nasal ridge point, two concave points of the maxillary zygomatic alveolar ridge on the coronal plane which bisect the apical plane of the first and second molars, one mesial contact point of incisors on the axial plane. The other eight points were cusps of left and right canines, buccal cusps of the first bicuspid, mesiobuccal cusps of the first molar, and mesiobuccal cusps of the second molars). The position deviation of miniscrews between the reconstructed 3D image of real miniscrews and the virtual image were then measured for deviation at the crown, and deviation at the apex. (Figure 8).

\section{Statistical analysis}

When implantation deviation was evaluated, the 


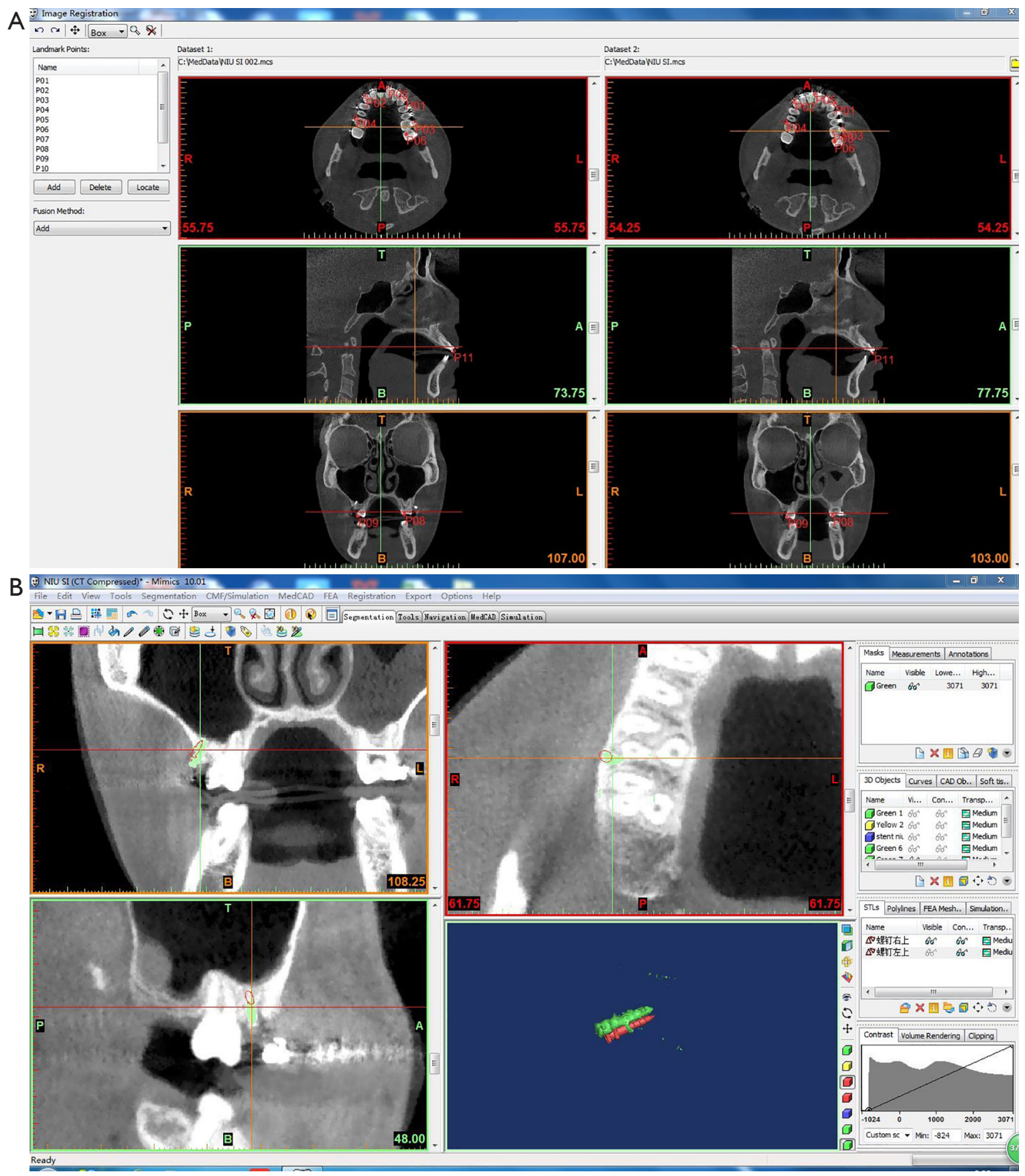

Figure 8 Implantation deviation evaluation. (A) Superimposition of pre- and post-implantation 3D images using 12 points. (B) Deviation in crown and apex.

superimposition and measurement were performed by three orthodontists with more than 3 years' experience in CBCT image analysis, and each measured once every week across three occasions. Descriptive analysis of quantitative data was performed and described with mean values, standard errors (SE), and $95 \%$ confidence intervals (95\% CIs). One way ANOVA was used to analyze whether there were statistical differences among the three measurements, and their average was considered as implantation deviation. 


\section{Maxillofacial changes after orthodontic treatment with template-guided implanted miniscrew anchorage}

Facial and occlusion photographs and lateral cephalometric radiographs were taken, and cast models were made before and after treatment to analyze treatment results with miniscrews as temporary anchorage.

\section{Results}

We implanted 24 miniscrews into the jaws of 10 patients under the guidance of a template. After implantation, cone beam CT showed that the miniscrews were not implanted into the maxillary sinus, and there was no contact with roots. Further, 18 miniscrews had a grade I safety score and six had a grade II. The mobility of miniscrews immediately after implantation was rated as level I, and all were stable at $1,3,6$, and 9 months after implantation, although there was mild inflammation around two miniscrews. Implantation deviation of 24 miniscrews was $(1.03 \pm 0.65) \mathrm{mm}$ in the crown and $(1.26 \pm 0.72) \mathrm{mm}$ in the apex, on average.

\section{Maxillofacial changes after orthodontic treatment}

All cases were Angle class II. Five cases were treated by extracting four first premolars, and five cases were treated by non-extraction. All cases were treated with miniscrew anchorage. The insertion sites in extraction first cases were between the maxillary lateral incisor and canine, and between the maxillary second bicuspid and first molar, while in non-extraction cases the insertion sites were between the first and second molars. Satisfactory occlusion and soft tissue results were acquired.

\section{Discussion}

Miniscrew implant anchorage is a powerful auxiliary device in orthodontic treatment, although the limited "safe area" in alveolar bone and jaws makes implantation difficult. Some studies have shown that serious dental pulp lesions occurred after orthodontic treatment for several years because of miniscrews penetrating or damaging the root (7). The goal of orthodontists is to provide more accurate, safe, and effective orthodontic treatment for patients. Therefore, how to improve the accuracy and safety of miniscrew assisted anchorage implantation and provide a reasonable and effective orthodontic force system to achieve good treatment results needs to be studied.
As mentioned, guided devices such as copper wire (12), 3D surgical guides (13), and graded 3D radiographic surgical guidance devices (14) can only offer 2D X-ray information and cannot provide adequate guidance to the $3 \mathrm{D}$ insertion area (15). Using 3D CT images combined with rapid prototyping and CAD-CAM technology, another surgical guide has been applied in clinical practice for prosthetic implants in recent years. Some research also showed surgical guides for miniscrew implantation in orthodontic treatment could be made with the same method or process, and the implantation accuracy was increased (16-20). However, in the former research (16), the author found the design and production system to be mostly suitable for prosthetic implants, which were placed in the edentulous area, while orthodontic miniscrews are commonly placed in the buccal or palatal alveolar septum. In addition, miniscrews are smaller and have a different shape to prosthetic implants, and the template of prosthetic implants has a larger volume and is more expensive. In some studies, spin-off slots on the insertion holes and guided cylinders were designed to remove surgical templates from patients $(19,20)$, and while this solved the problem of removing the template after implantation, it could result in inadequate guidance during implantation. In our study, we improved the surgical template design and production system to make the computer design process simpler, and to make the guide more accurate and suitable for orthodontic miniscrew use. 3D virtual images of orthodontic miniscrews were imported into the system to make the virtual implantation more simulated. The registration process of CBCT images of bone and teeth with laser scanning images of dentition and mucosa were abandoned, making the process simpler and reducing the registration error. The retention part was fitted in with the teeth, not the mucosa, and close to the insertion site, and the guide portion was a cylindrical tube and kept a certain distance from the mucosa of the insertion site. In this process, the distance should be shorter than the length of screwdriver's cylindrical tip, and this distance, plus the thickness of the guide part, should be longer than the length of the miniscrew body. This aims to ensure the screwdriver tip carrying the miniscrew can be adequately guided in the whole implantation process, the mucosa condition in the insertion area can be visible in the template-guided implantation process to detect oral mucosa folding and accumulation during implantation, and the guide template can be freely taken off after the implantation without the interference of the exposed crown part of miniscrew.

The design and production process of guide plates 
took the following steps: Firstly, on consideration of the treatment purpose, the orthodontic force system to be applied, and the local condition of bone and the root evaluated with CBCT images, the insertion position was determined. The position and direction of the virtual miniscrews were then adjusted in Mimics software, to avoid possible damage to the anatomical structure of the insertion area, such as touching the root, and penetrating the maxillary sinus and the nerve canal. After the position and direction of the miniscrews were determined, the $3 \mathrm{D}$ design of the guide template was carried out, so that the position relationship between the jaw and the miniscrews was fixed with the template. Finally, the real template was printed by stereolithography $3 \mathrm{D}$ printing technology. The retention part of the guide template ensured that the guide template was stably located on the teeth close to the insertion area, the guide part could guide the screwdriver tip carrying the miniscrew to implant it into the predetermined position, and the connecting part connected the guide part and the retention part.

Before use, the template was placed on a cast model to ensure its fit and stability, and when transferred to the patient's mouth, the patient was instructed to bite gently, or the operator applied light force on the retention part of the template to make it stable.

The clinical evaluation showed that the 24 miniscrews implanted under the guidance of the template did not contact the root, the maxillary sinus, or other important anatomical structures. Postoperative CBCT analysis also showed that all screw placements were grade I and II and that any deviations were acceptable. Only three sites had mild inflammation around the miniscrews, which may be related to poor oral hygiene, or that the insertion sites were apically to the mucogingival junction. All 24 miniscrews were stable during the orthodontic treatment.

Most studies that have investigated template-guided implantation for prosthetic implants have shown similar levels of accuracy. In a study conducted by Schneider et al. (21), the mean error was $1.07 \mathrm{~mm}$ at the coronal center and $1.63 \mathrm{~mm}$ at the apical center, and in a systematic review performed by Van Assche et al. (22), the mean error was $0.99 \mathrm{~mm}$ at the coronal center, $1.24 \mathrm{~mm}$ at the apical center. Compared to other studies of template guided implantation, the deviation of miniscrews in our study were similar to those of the previously presented systematic reviews.

Deviation of miniscrew position can come from errors in several components of the process including image acquisition and data processing error, superimposition error of the CBCT image with cast laser image, errors in the surgical template fabrication process with stereolithography, tolerance between the guide channel and drill or driver, inadequate template positioning, and template movement during implantation. Loss of accuracy can occur from any of these alone or in combination. In our study, we used a tooth supporting template, deleted the fusion process, reduced the tolerance between the guide tube and screwdriver tip, and designed a certain distance gap to ensure adequate guidance during the whole implantation process.

It showed that miniscrew implantation safety and stability were sound when using this template. We also suggest that insert the miniscrew in the direction of $30^{\circ}$ to $45^{\circ}$ angulation to the tooth axial in the posterior area of dentition, at the horizontal level of mucogingival junction.

After being implanted in the guide of surgical templates, miniscrews were used as anchorage to correct malocclusion. Five cases of skeletal Class II high angle extraction and five cases of class II non extraction were treated. Miniscrews implanted between the maxillary lateral incisors and canines aimed to intrude the anterior teeth, and those between the maxillary second premolars and first molars were used to control the posterior teeth sagittally and vertically. Miniscrews were implanted between the maxillary first and second molars in non-extraction cases which needed molar and arch distal movement.

Cephalometric and cast models before and after treatment were measured to analyze the dentomaxillofacial changes of patients in force system with orthodontic miniscrews as anchorage. The $3 \mathrm{D}$ changes of Class II high angle extraction patients were as follows: in the sagittal direction, Class I occlusion were acquired, anterior overbite and arch length were reduced, and the maxillary incisors were retracted. Maxillary molars had no obvious mesiodistal movement, which means good control of the upper molar was obtained, while mesial movement of the mandibular molars was also found. In the transversal direction, both upper and lower arches had no significant changes, and in the vertical direction, the maxillary molars were intruded slightly, and the upper and lower anterior teeth were intruded. The nasolabial angle of soft tissue increased, the distance from the upper and lower lip to the $\mathrm{G}$ line shortened, and the facial tissue profile was also improved. In non-extraction cases, the treatment results were as follows: Class I occlusion was acquired, the width of the canine increased slightly, the width of the bicuspid and molar increased significantly, and the length of the dental arch decreased. The maxillary molar moved distally. All 
cases acquired sound treatment results and retained stable. The chief limitation of this study is its small sample size and more patients could be enrolled in the future study to verify the results. The use of a greater number of virtual miniscrews to facilitate the design and manufacture system of the template should be included, and more 3D design software could be used.

\section{Conclusions}

A novel CBCT image-based CAD-CAM template for orthodontic miniscrew implantation was introduced. When guided by the CAD-CAM template, miniscrews can be safely and accurately implanted into the determined position and kept stable to achieve satisfactory orthodontic treatment results.

\section{Acknowledgments}

We thank all co-authors for support from data collection to manuscript revision.

Funding: This work was supported by the Natural Science Foundation of China (No. 81400567), Beijing Natural Science Foundation (No. 7132056), and "Beijing Hospitals Authority" Ascent Plan (DFL20191501). The content is solely the responsibility of the authors and does not necessarily represent the official views of the NSFC and BNSF.

\section{Footnote}

Reporting Checklist: The authors have completed the STROBE reporting checklist. Available at https://dx.doi. org/10.21037/atm-21-2575

Data Sharing Statement: Available at https://dx.doi. org/10.21037/atm-21-2575

Conflicts of Interest: All authors have completed the ICMJE uniform disclosure form (available at https://dx.doi. org/10.21037/atm-21-2575). All authors report that this work was supported by the Natural Science Foundation of China (No. 81400567), Beijing Natural Science Foundation (No.7132056), and "Beijing Hospitals Authority" Ascent Plan (DFL20191501). The authors have no other conflicts of interest to declare.

Ethical Statement: The authors are accountable for all aspects of the work in ensuring that questions related to the accuracy or integrity of any part of the work are appropriately investigated and resolved. The ethics committee of Beijing Stomatological Hospital approved the study protocol (2015 No. 55), which was conducted in full accordance with ethical principles, including the World Medical Association Declaration of Helsinki (as revised in 2013). All patients included in this study signed an informed consent for the scientific use of data.

Open Access Statement: This is an Open Access article distributed in accordance with the Creative Commons Attribution-NonCommercial-NoDerivs 4.0 International License (CC BY-NC-ND 4.0), which permits the noncommercial replication and distribution of the article with the strict proviso that no changes or edits are made and the original work is properly cited (including links to both the formal publication through the relevant DOI and the license). See: https://creativecommons.org/licenses/by-nc-nd/4.0/.

\section{References}

1. Chang CH, Lin LY, Roberts WE. Orthodontic bone screws: A quick update and its promising future. Orthod Craniofac Res 2021;24 Suppl 1:75-82.

2. Poggio PM, Incorvati C, Velo S, et al. "Safe zones": a guide for miniscrew positioning in the maxillary and mandibular arch. Angle Orthod 2006;76:191-7.

3. Baumgaertel S, Hans MG. Buccal cortical bone thickness for miniimplant placement. Am J Orthod Dentofacial Orthop 2009;136:230-5.

4. Murugesan A, Sivakumar A. Comparison of bone thickness in infrazygomatic crest area at various miniscrew insertion angles in Dravidian population - A cone beam computed tomography study. Int Orthod 2020;18:105-14.

5. Parmar R, Reddy V, Reddy SK, et al. Determination of soft tissue thickness at orthodontic miniscrew placement sites using ultrasonography for customizing screw selection. Am J Orthod Dentofacial Orthop 2016;150:651-8.

6. Lim G, Kim KD, Park W, et al. Endodontic and surgical treatment of root damage caused by orthodontic miniscrew placement. J Endod 2013;39:1073-7.

7. Er K, Bayram M, Taşdemir T. Root canal treatment of a periradicular lesion caused by unintentional root damage after orthodontic miniscrew placement: a case report. Int Endod J 2011;44:1170-5.

8. Sfondrini MF, Gandini P, Alcozer R, et al. Failure load and stress analysis of orthodontic miniscrews with different transmucosal collar diameter. J Mech Behav Biomed Mater 
2018;87:132-7.

9. Kuroda S, Tanaka E. Risks and complications of miniscrew anchorage in clinical orthodontics. Jpn Dent Sci Rev 2014;50:79-85.

10. Ahmed VK, Krishnaswamy NR, Thavarajah R. Miniscrew implant fracture and effects of such retained tip on dentinpulp complex: a histological report. Dent Traumatol 2016;32:161-5.

11. Papageorgiou SN, Zogakis IP, Papadopoulos MA. Failure rates and associated risk factors of orthodontic miniscrew implants: a meta-analysis. Am J Orthod Dentofacial Orthop 2012;142:577-95.e7.

12. Sharma K, Sangwan A. K.s. Micro-implant placement guide. Ann Med Health Sci Res 2014;4:S326-8.

13. Suzuki EY, Suzuki B. Accuracy of miniscrew implant placement with a 3-dimensional surgical guide. J Oral Maxillofac Surg 2008;66:1245-52.

14. Estelita S, Janson G, Chiqueto K, et al. Predictable drillfree screw positioning with a graduated 3-dimensional radiographic-surgical guide: a preliminary report. Am J Orthod Dentofacial Orthop 2009;136:722-35.

15. Miyazawa K, Kawaguchi M, Tabuchi M, et al. Accurate pre-surgical determination for self-drilling miniscrew implant placement using surgical guides and cone-beam computed tomography. Eur J Orthod 2010;32:735-40.

16. Qiu L, Haruyama N, Suzuki S, et al. Accuracy of orthodontic miniscrew implantation guided by stereolithographic surgical stent based on cone-beam CT- derived 3D images. Angle Orthod 2012;82:284-93.

17. Cassetta M, Altieri F, Giorgio RD, Barbato E. Palatal orthodontic miniscrew insertion using a CAD-CAM surgical guide: description of a technique. Int J Oral Maxillofac Surg 2018;47:1195-8.

18. Bae MJ, Kim JY, Park JT, et al. Accuracy of miniscrew surgical guides assessed from cone-beam computed tomography and digital models. Am J Orthod Dentofacial Orthop 2013;143:893-901.

19. Wang Y'T, Yu JH, Lo LJ, et al. Developing Customized Dental Miniscrew Surgical Template from Thermoplastic Polymer Material Using Image Superimposition, CAD System, and 3D Printing. Biomed Res Int 2017;2017:1906197.

20. Yu JH, Wang Y'T, Lin CL. Customized surgical template fabrication under biomechanical consideration by integrating CBCT image, CAD system and finite element analysis. Dent Mater J 2018;37:6-14.

21. Schneider D, Marquardt P, Zwahlen M, et al. A systematic review on the accuracy and the clinical outcome of computer-guided template-based implant dentistry. Clin Oral Implants Res 2009;20 Suppl 4:73-86.

22. Van Assche N, Vercruyssen M, Coucke W, et al. Accuracy of computer-aided implant placement. Clin Oral Implants Res 2012;23 Suppl 6:112-23.

(English Language Editor: B. Draper)
Cite this article as: Qiu L, Xu H, Feng P, Sha X, Zhang H. Clinical effectiveness of orthodontic miniscrew implantation guided by a novel cone beam CT image-based computer aided design and computer aided manufacturing (CAD-CAM) template. Ann Transl Med 2021;9(12):1025. doi: 10.21037/atm-212575 